\title{
Non-proportionality and Photon Interaction Study of CLYC Scintillation Material by Compton Scattering Technique
}

\author{
Wuttichai Chaiphaksa ${ }^{1}$, Wiraporn Hongtong ${ }^{2}$, Wasu Cheewasukhanont ${ }^{3}$, Kittipong \\ Siengsanoh $^{4}$, Jakrapong Kaewkhao ${ }^{5}$ \\ \{wuttichai.chaiphaksa@gmail.com ${ }^{1}$ \} \\ Physics Program, Faculty of Science and Technology, Nakhon Pathom Rajabhat University ${ }^{1}$, Center \\ of Excellence in Glass Technology and Materials Science (CEGM), Nakhon Pathom Rajabhat \\ University ${ }^{2,3,4,5}$
}

\begin{abstract}
In this research, scintillation properties of $\mathrm{Cs}_{2} \mathrm{LiYCl}_{6}: \mathrm{Ce}$ (CLYC) scintillation material were studied by Compton scattering technique and the samples were irradiated with eight different photon energies using these technique. Photon interactions have been calculated by WinXCom program at 216-662 keV. The mass attenuation

coefficient $\left(\mu_{m}\right)$ and partial interactions, light yield and non-proportionality were presented. The values of increased toward the decrease of gamma ray energies and are influenced by photon energy and chemical compositions. The partial interactions found that three energy relative to the partial processes first photoelectric absorption, second incoherent (Compton) scattering and third coherent (Rayleigh) scattering.Moreover, the crystal were higher at the low photon energy and decreased with increasing the photon energy. The results of scintillation properties show that slightly increased when increasing the photon energy.The non-proportionality shows demonstrated excellent proportional property in the energy range, the non-proportionality is about $1.5 \%$.
\end{abstract}

Keywords: scintillation material, non-proportionality, mass attenuation coefficient, light yield.

\section{Introduction}

In the present, scintillation material are most widely used for the detection of X-rays, gamma rays, and thermal neutrons. Moreover, many type of the scintillation material were among the most popular to applied in nuclear physics laboratories. (Van Eijk, 2001)In 1999, the researchers from Delf University of Technology was first discovered a new inorganic scintillator cerium-doped cesium lithium yttrium chloride (CLYC). The CLYC crystal was the first applied gamma \& neutron scintillation detector for use as a replacement for both medium resolution gamma-ray detectors and helium-3 proportional counter tubes for neutron detection. (Qin, 2018)The background ofCLYC crystal is very good energy resolution (better than 5\% FWHM at $662 \mathrm{keV}$ ), the density is $3.31 \mathrm{~g} / \mathrm{cm} 3$ and peak scintillation wavelength is $370 \mathrm{~nm}$. The ideal scintillation material should convert the kinetic energy of the charged particles or gamma ray into detectable light with high scintillation efficiency. The light yield should be proportional to the deposited energy over a possible wide range.But, still no material meets all 
these criteria. (Flakus, 1980,Limkitjaroenporn, 2010)Under these conditions, fundamental properties of scintillation material are very important for example mass attenuation coefficient, decay time, energy resolution,light yield and non-proportionality of light yield. (Moszynski, 2010)

In this study, to investigate scintillation crystal of CLYC for gamma ray spectroscopy. The following properties will be studied on the mass attenuation coefficient, light yield and non-proportional of light yield. The ${ }^{137} \mathrm{Cs}$ have been used in this experiment and using Compton scattering technique for varying gamma ray energies.

\section{Experiment}

The study of CLYC scintillator with the size of $12.7 \times 12.7 \times 12.7 \mathrm{~mm}^{3}$, the crystal were measured with a photomultiplier tube (PMT) number $9256 \mathrm{~KB}$ by ET Enterprise electron tubes.

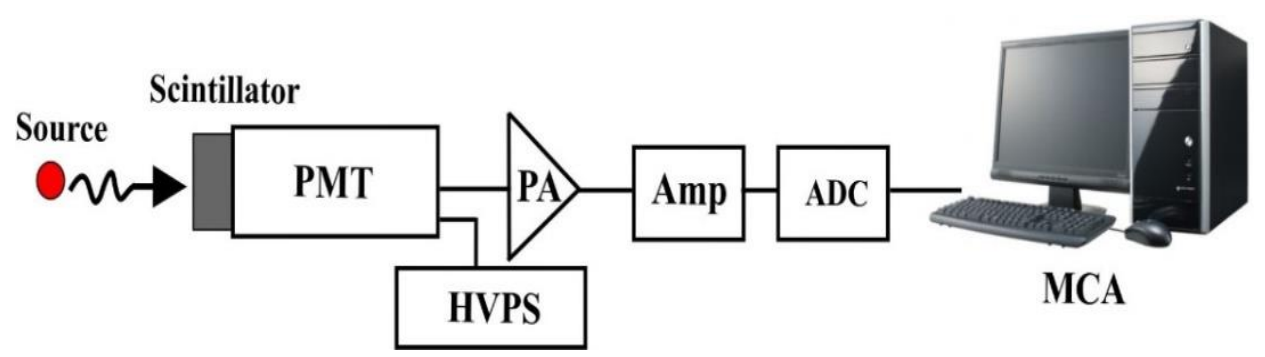

Fig.1. Schematic representation of the experimental setup used to measure photoelectron yield and light yield. 


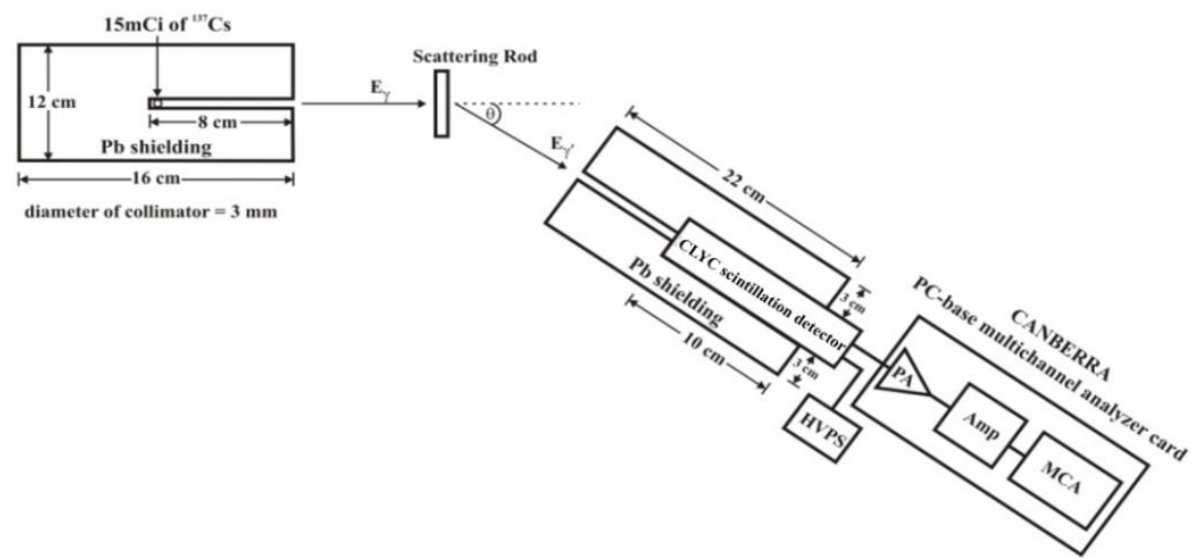

Fig.2.Block diagram of the Compton scattering technique for the measurement of photoelectron yield and light yield.

Table 1.The scattered gamma rays energies at different angles

\begin{tabular}{cccc}
\hline$\Theta(\operatorname{deg})$ & $\mathrm{E}_{\gamma(\mathrm{th})}(\mathrm{keV})$ & $\mathrm{E}_{\gamma(\mathrm{ex})}(\mathrm{keV})$ & $\% \mathrm{RD}$ \\
\hline 30 & 564.09 & $573.85 \pm 13.75$ & 1.72 \\
45 & 479.90 & $486.67 \pm 10.25$ & 1.41 \\
60 & 401.76 & $399.50 \pm 8.16$ & 0.56 \\
75 & 337.72 & $346.15 \pm 21.17$ & 2.49 \\
90 & 288.39 & $281.10 \pm 22.03$ & 2.52 \\
105 & 251.63 & $245.97 \pm 28.35$ & 2.25 \\
120 & 224.92 & $216.95 \pm 24.32$ & 3.21 \\
\hline
\end{tabular}


A photomultiplier tube coupled the crystals to using silicone grease covered with several layers of white teflon tape and wrapped with black tape, then covered with aluminum housing. The signal output from the PMT anode passed through a Canberra 2007B preamplifier and was sent to Canberra 2022 an amplifier set at $0.5 \mu \mathrm{s}$ a shaping time constant. The energy spectra analysed with software of Canberra MCA to record data. The measurements were carried out with 30 of CLYC scintillator in the amplifier. The high voltage power supply (HVPS) was used negative $620 \mathrm{~V}$ for CLYC scintillators as shown in figure 1 .

Figure 1 and figure 2 shows the experimental setup and block diagram used to measure photoelectron yield and light yield using Compton scattering technique for varying gamma ray energies. The ${ }^{137} \mathrm{Cs}$ obtained from the Office of Atom for Peace (OAP), Thailand, with an activity of $15 \mathrm{mCi}$ (555 $\mathrm{MBq})$. The relation of the energy transfer and the scattering angle for any given interaction can be expressed from simultaneous equations for the conservation of energy and momentum (Glenn, 2000). The Compton scattering requires that the light is viewed as a particle and not just a wave because it is the collision of photon with electron and the exchange of energy, which accounts for the shift in energy. The energy imparted to the recoil electron is given by Compton (Tsoulfanidis, 1995) according to the equation:

$$
E_{\gamma}^{\prime}=\frac{E_{\gamma}}{1+(1-\cos \theta) E_{\gamma} / m_{0} c^{2}}
$$

where $E_{\gamma}^{\prime}$ is the scattered gamma ray energy, $E_{\gamma}^{\prime}$ is the incident gamma ray energy, $\theta$ is the scattering angle and is the rest-mass-energy of the electron $(551 \mathrm{keV})$.

To measure the mass attenuation coefficient, light yield and non-proportional of light yield, the Compton scattering technique was applied at seven different angles $(\Theta)$ from $30^{\circ}$ $120^{\circ}$ using Compton scattering technique to produce $\gamma$-rays of different energies. The validity of the system has been confirmed with an energy calibration of the system. It was found that seven corresponding photon energies were in the range of $216-662 \mathrm{keV}$ as shown in Table 1.

\section{Results And Discussion.}

\subsection{Mass Attenuation Coefficient and Partial Interactions}

Theoretically, the mass attenuation coefficient of CLYC crystal compared with BGO single crystal by WinXCom program are shown in Figure 3. It is seen that mass attenuation coefficient are influenced by photon energy and chemical compositions. It was indicates that, at lower energies the total photon interactions with both scintillator is high while at higher energies it decreases. It is clearly to observed that the mass attenuation coefficient of BGO crystal have higher values at lower energies than CLYC crystal. Due to the BGO crystal is very high density $\left(7.11 \mathrm{~g} / \mathrm{cm}^{3}\right)$ and effective atomic number.

Figure 4 shows the partial interaction of CLYC crystal, it was found that three energy relative to the partial processes first photoelectric absorption has shown to be a stronger effect at low energy, second incoherent (Compton) scattering is the main interaction in the Compton energy range and third coherent (Rayleigh) scattering it is not significant role in this connection because the value was negligible and decreases almost zero at high energies. 


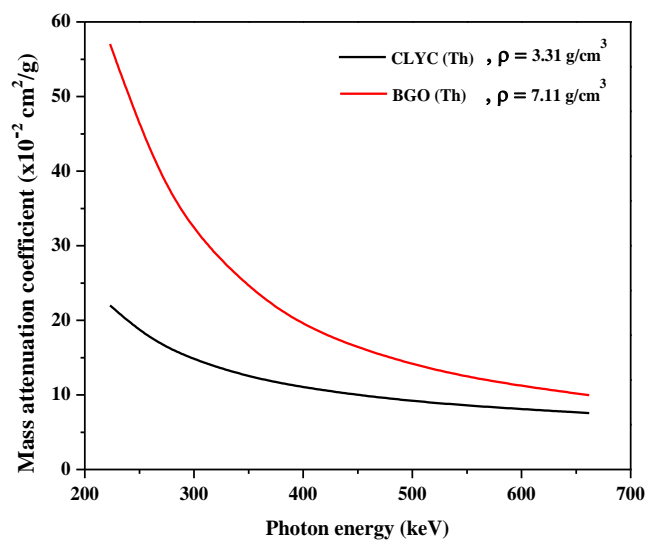

Fig. 3. The mass attenuation coefficient of CLYC crystal compared with BGO single crystal at 216-662 $\mathrm{keV}$.

Figure 5 shows the number of photoelectron (Nphe) of CLYC crystal at 216-662 keV, this value is about when the crystal interaction with photon energy and emitted the light per unit area per unit time which directly proportional to the intensity of light used. The results show that these value response linear with the photon energy. The Nphe is very important to transform the number of photoelectron per $\mathrm{MeV}(\mathrm{Nphe} / \mathrm{MeV})$ into an absolute light yield $(\mathrm{Ph} / \mathrm{MeV})$ as shown in figure 6.

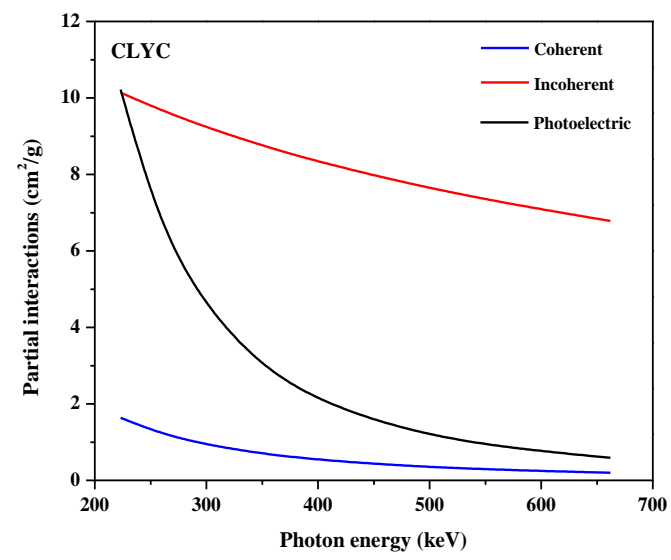

Fig. 4. The partial interactions of CLYC crystal at 216-662 keV. 


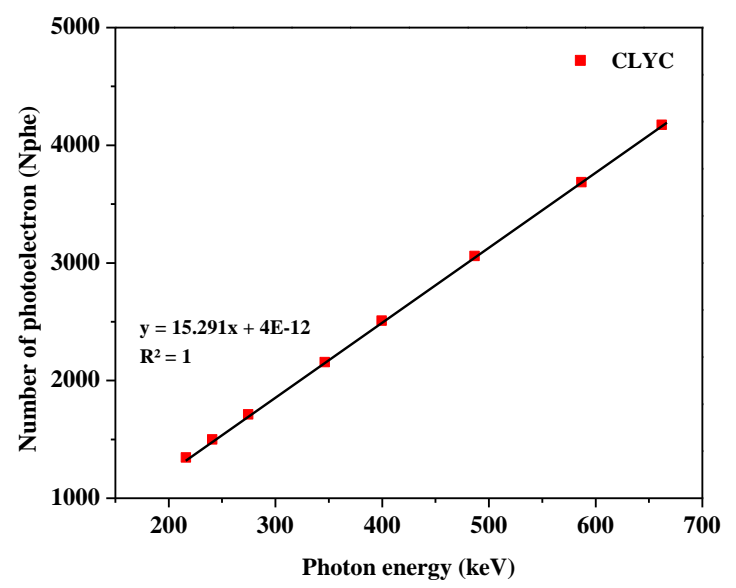

Fig. 5. The number of photoelectron of CLYC crystal at 216-662 keV.

From figure 6, the light yield measurement procedure as determined by Bertolaccini method (Bertolaccini, 1968) and the non-proportionality of CLYC crystal in the energy range of $216-662 \mathrm{keV}$ are shown in figure 7 . It is seen that the plots of the relationship between the light yield and energy slightly increased when increasing the photon energy. The nonproportionality of CLYC crystal normalized at $662 \mathrm{keV}$ shows demonstrated excellent proportional property in the energy range, the non-proportionality is about $1.5 \%$.

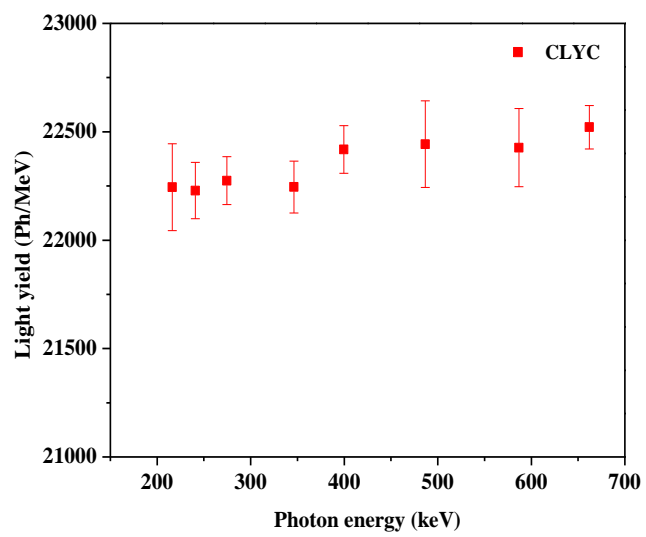

Fig. 6.The light yield of CLYC crystal at 216-662 keV. 


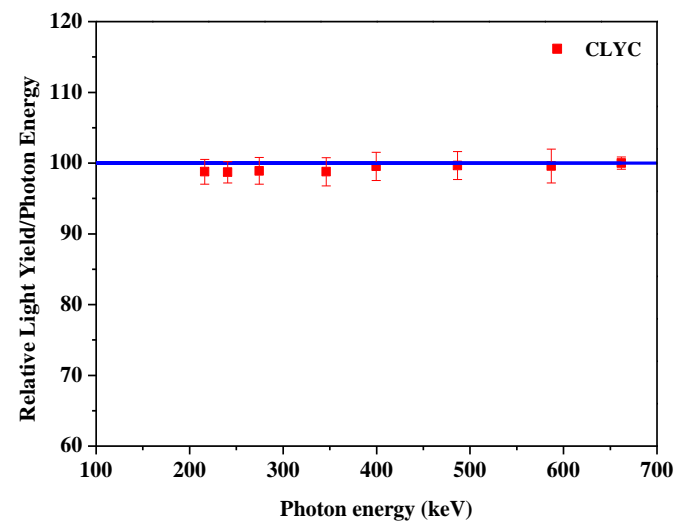

Fig.7.The non-proportional of light yieldof CLYC crystal at 216-662 keV

\section{Conclusions}

The scintillation properties of CLYC crystal using Compton scattering technique was finished measurement. It was found that seven corresponding photon energies were in the range of 216-662 keV. Photon interactions have been calculated by WinXCom program at $216-662 \mathrm{keV}$. The mass attenuation coefficient $\left(\mu_{m}\right)$ and partial interactions, light yield and non-proportionality were presented. The values of $\left(\mu_{m}\right)$ increased toward the decrease of gamma ray energies and $\left(\mu_{m}\right)$ are influenced by photon energy and chemical compositions. The partial interactions found that three energy relative to the partial processes first photoelectric absorption, second incoherent (Compton) scattering and third coherent (Rayleigh) scattering. Moreover, the crystal were higher at the low photon energy and decreased with increasing the photon energy. The results of scintillation properties show that slightly increased when increasing the photon energy. The non-proportionality shows demonstrated excellent proportional property in the energy range, the non-proportionality is about $1.5 \%$.

Acknowledgements. The authors gratefully acknowledge the National Research Council of Thailand (NRCT) for supporting this research and Nakhon Pathom Rajabhat University (NPRU) for facilities.

\section{References}

[1]Bertolaccini, M., Cova, S., Bussolatti, C., A technique for absolute measurement of the effective photoelectron per $\mathrm{keV}$ yield in scintillation counters, In the Proceeding of the Nuclear Electronics Symposium (10-13 September). Versailles, France: n.p. 1968.

[2]Flakus, F. N., Detecting and measuring ionizing radiation - a short history. IAEA BULLETIN, 23, pp. 31-36, 1980. 
[3]Limkitjaroenporn, P., et al. Nonproportionality of electron response using CCT: Plastic scintillator. Applied Radiation and Isotopes, 68, pp. 1780-1784, 2010.

[4]Moszynski, M., Energy resolution and non-proportionality of scintillation detectors new observations, Radiation Measurements, vol. 45, pp. 372-376, 2010.

Qin, J., et al. Characterization of a 6Li enriched Cs2LiYCl6:Ce scintillator and its application as a $\square$-ray detector, Nuclear Inst. and Methods in Physics Research A, vol. 886, pp. 55-60, 2018.

[5]Tsoulfanidis, N., Measurement and detection of radiation, Washington, DC.: Taylor \& Francis, 2nd edition, 1995.

[6]Van Eijk, C.W.E. Inorganic-scintillator development, Nuclear Instruments and Methods in Physics Research A, vol. 460, pp. 1-14, 2001. 